\title{
Mapping the transition to superwetting state for nanotextured surfaces templated from block-copolymer self-assembly
}

Telecka, Agnieszka; Mandsberg, Nikolaj Kofoed; Li, Tao; Ludvigsen, Emil; Ndoni, Sokol; Di Mundo, Rosa; Palumbo, Fabio; Fiutowski, Jacek; Chiriaev, Serguei; Taboryski, Rafael

\section{Published in:}

Nanoscale

Link to article, DOI:

10.1039/c8nr07941b

Publication date:

2018

Document Version

Peer reviewed version

Link back to DTU Orbit

Citation (APA):

Telecka, A., Mandsberg, N. K., Li, T., Ludvigsen, E., Ndoni, S., Di Mundo, R., Palumbo, F., Fiutowski, J., Chiriaev, S., \& Taboryski, R. (2018). Mapping the transition to superwetting state for nanotextured surfaces templated from block-copolymer self-assembly. Nanoscale, 10(44), 20652-20663.

https://doi.org/10.1039/c8nr07941b

\section{General rights}

Copyright and moral rights for the publications made accessible in the public portal are retained by the authors and/or other copyright owners and it is a condition of accessing publications that users recognise and abide by the legal requirements associated with these rights.

- Users may download and print one copy of any publication from the public portal for the purpose of private study or research.

- You may not further distribute the material or use it for any profit-making activity or commercial gain

- You may freely distribute the URL identifying the publication in the public portal 


\section{Mapping the transition to superwetting state for nanotextured surfaces templated from}

\section{block-copolymer self-assembly}

Agnieszka Telecka1,\#, Nikolaj K. Mandsberg ${ }^{1, \#}$, Tao Li ${ }^{1,2}$, Emil Ludvigsen ${ }^{1}$, Sokol Ndoni ${ }^{1,3}$, Rosa Di Mundo ${ }^{4}$, Fabio Palumbo ${ }^{5}$, Jacek Fiutowski ${ }^{6}$, Serguei Chiriaev ${ }^{6}$, and Rafael Taboryski ${ }^{1, *}$

${ }^{1}$ Department of Micro- and Nanotechnology, Technical University of Denmark, DK-2800 Kongens Lyngby, Denmark

${ }^{2}$ Department of Electronic and Electrical Engineering, University College London, WC1E7JE London, UK

${ }^{3}$ Center for Nanostructured Graphene, CNG, Technical University of Denmark, DK-2800 Kongens Lyngby, Denmark

${ }^{4}$ Department of Chemistry (DICATECh), Politecnico di Bari, via Orabona 4, 70126 Bari, Italy

${ }^{5}$ Institute of Nanotechnology (NANOTEC), National Research Council, 70126 Bari, Italy

${ }^{6}$ Mads Clausen Institute, University of Southern Denmark, DK-6400 Sønderborg, Denmark

*rata@nanotech.dtu.dk

\#Agnieszka Telecka and Nikolaj K. Mandsberg contributed equally to this work. 


\begin{abstract}
Adding roughness to hydrophilic surfaces is generally expected to enhance their wetting by water. Indeed, global free energy minimization predicts decreasing contact angles when roughness factor or surface energy increases. However, experimentally it is often found that water spreading on rough surfaces is impeded by pinning effects originating from local free energy minima; an effect, largely neglected in scientific literature. Here, we map the transition to a superwetting state of hydrophilic nano-textured surfaces in terms of surface chemistry and texture geometry by utilizing Laplace pressure as a proxy for these local minima. We demonstrate the effect for polymer model surfaces templated from block-copolymer self-assembly comprising dense, nano-pillar arrays exhibiting strong pinning in their pristine state. By timed argon plasma exposure, we tune surface chemistry to map the transition into the superwetting state of low contact angle, which we show coincide with the surface supporting hemiwicking flow. For the near-ideal model surfaces, the transition to the superwetting state occurs below a critical material contact angle of $\sim 50^{\circ}$. We show that superwetting surfaces possess anti-fogging properties, and demonstrate long term stability of the superwetting effect by coating the nanotextured surfaces with $\sim 10 \mathrm{~nm}$ thin films of either tungsten or silica.
\end{abstract}

Keywords: superwetting, hemiwicking, antifogging, block-copolymer self-assembly, nanofabrication, plasma treatment, silicon oxide, tungsten. 


\section{Introduction}

Designing the wetting properties of materials is central to a broad range of industries, notably medicine, optics, and energy, and has consequently spawned huge research efforts in the last two decades. ${ }^{1}$ Fortunately, Nature has developed a vast range of intriguing wetting properties readily solving major challenges. Examples include hierarchically structured self-cleaning surfaces inspired by the Lotus leaf, ${ }^{1-4}$ as well as surfaces to control dropwise condensation, ${ }^{5-8}$ ice formation, ${ }^{8-10}$ and fogging ${ }^{11,12}$. Such surfaces can alleviate issues with fogging of lenses, mirrors, goggles, and wind shields; ${ }^{13}$ reduction of sunlight harvesting in photovoltaic devices; ${ }^{13,14}$ and poor field visibility in endoscopy ${ }^{15}$. For the wetting or de-wetting performance of these engineered surfaces, the pinning effect is often encountered as a central problem. ${ }^{16}$ In terms of the traditional energy minimization approach to describe wetting, ${ }^{17}$ pinning impedes droplets in reaching the global minimum of Gibbs free energy, characterized by either a pure Wenzel ${ }^{18}$ or a pure Cassie-Baxter ${ }^{19}$ state. For both hydrophilic and hydrophobic surfaces, pinning is also responsible for the contact angle hysteresis, ${ }^{3,}$ $16,20,21$ by trapping droplets in metastable states, ${ }^{3}$ often requiring some agitation of the triple line to overcome the barriers induced by pinning. ${ }^{16,22,23}$

Recently, Mouterde et al. showed how the fogging of hydrophobic structured surfaces is significantly decreased when mimicking the nanotextures of cicada (Psaltoda claripennis) wings. ${ }^{12}$ They attributed the enhanced antifogging effect to a reduced adhesion of micro droplets when textures are downsized to nanoscale. While Mouterde et al. and most other studies are concerned with superhydrophobic surfaces, the antifogging effect for nanoscale-textured, hydrophilic surfaces remains largely unexplored despite a huge potential for applications such as fogharvesting ${ }^{24}$ and oil-water separation ${ }^{25}$. Much attention is also currently given to photo-induced 
wettability conversion of metal oxide thin films, where intrinsically hydrophilic materials exhibit very high contact angles. ${ }^{26}$ However, few, if any, studies go into details with the reason for this abnormal contact angle behaviour.

A "superwetting surface" is sometimes understood as synonymous to a "superhydrophilic surface", i.e. a surface that exhibits a contact angle to water lower than the Young contact angle for the same material. ${ }^{27}$ Another definition is: any surface that will spread a droplet to a thin film. ${ }^{28}$ Here, we define superwetting as a type of wetting that allows droplets to spread on the surface with no pinning. The main novel finding presented here is that a unified understanding of the two definitions above can be obtained by looking into the coincident conditions for spontaneous hemiwicking flow ${ }^{29}$ and fast spreading of droplets. This implies that superwetting of nanotextured surfaces is closely related to their ability to support spontaneous hemiwicking; i.e. flow in-between the open surface textures driven by uncompensated Laplace pressure for the advancing liquid menisci; ${ }^{30-32}$ contrary, surfaces not supporting hemiwicking will tend to pin droplets. The Laplace pressure, which may either act as a driving force or a barrier for the flow, is of order $\gamma / \zeta$, where $\gamma$ is the surface tension of the liquid, and $\zeta$ is the characteristic pore size. The barrier properties of the Laplace pressure are well known from microfluidic capillary-stop devices, ${ }^{33}$ and from omniphobic surfaces based on re-entrant texture geometries ${ }^{34}$. If $\zeta$ is downsized to nanoscale, this Laplace pressure may compare to hundreds of meters hydrostatic pressure.

The technologically interesting implication of the reported wetting transition to the superwetting state is that a surface exposed to fog will form a continuous water film. For such fully wetted textured surfaces, optical transparency under condensation is preserved due to replacement of 
the myriads of light-scattering micro-droplets by the water film. In addition, we note that texture sizes must be below the diffraction limit of light to avoid haze, ${ }^{35}$ which for visible light is $\sim 200$ $\mathrm{nm} .{ }^{36}$ Hence, we report on the hemiwicking and antifogging properties of nanotextured model surfaces templated from block-copolymer self-assembly with pillar diameters of $\sim 50 \mathrm{~nm}$ arranged in a predominantly hexagonal array with pitch size of $\sim 70 \mathrm{~nm} .{ }^{37,}{ }^{38}$ Structures with an aspect ratio $\sim 1$ are replicated in highly transparent poly(methyl methacrylate) (PMMA), which allows study of the $\sim 50 \mathrm{~nm}$ thick wicking films by transmission microscopy ${ }^{39}$. On the microscale, antifogging properties are studied by controlled condensation of water vapor ${ }^{7}$ on the surfaces.

\section{Results and discussion}

We experimentally demonstrate that spontaneous hemiwicking is a critical condition for macroscopic superwetting by developing a method to fine-tune the surface energy of the substrate. The results are shown in Figure 1. Figure 1a shows the measured water contact angles for unstructured and structured sample siblings that underwent simultaneous argon plasma treatment. To avoid plasma-induced roughness, mild plasma were employed and allowed an exclusively chemical modification. Continuous tuning of the surface energy was easily achieved by plasma time variation, thus allowing the observation of droplet-transition between the two wetting regimes. The data points marked with $\mathbf{A}^{\prime}$ and $\mathbf{B}^{\prime}$, represent, respectively, a surface with severe pinning (no plasma treatment) and clear superwetting resulting from a $70 \mathrm{~s}$ Ar plasma treatment. One immediate observation for condition $\mathbf{A}^{\prime}$ is that the contact angle for the nanotextured sample is higher than the corresponding Young contact angle; measured on its flat sample sibling (Figure 1b). On the other hand, for sample siblings subjected to the plasma 
treatment, the contact angle of the nanotextured sample is well below the Young contact angle ${ }^{40}$; measured on corresponding unstructured/flat samples (Figure 1c). The increased contact angles are observed for the nano-structured, but untreated, samples despite their intrinsic hydrophilicity. Again, from a global energy minimization perspective the roughness should have increased the wettability. This qualitative difference between the two regimes is also present at microscale as seen in Figures 1d,e. Here, we show fogging experiments for the two extreme samples $\mathbf{A}^{\prime}$ and $\mathbf{B}^{\prime}$, performed in an environmental chamber featuring reproducible condensation of water vapor (see Methods section). For comparison, the fogging experiments were also carried out using the corresponding flat samples. We see a clear difference between the nanostructured and flat samples; only when structure is present, and surface chemistry is favorable, the sample exhibit full wetting of the entire surface. Although the condensation time depends on substrate cooling, full wetting was here obtained after condensation for 25 minutes, and no further change in the wetting morphology happened hereafter. To illustrate the improvement for visual applications, we tested the fogging properties for the entire $50 \mathrm{~mm}$ samples by exposing them to hot water vapor (Figures 1f, 1g). Again, we present the two extreme cases $\left(\mathbf{A}^{\prime}\right.$ and $\left.\mathbf{B}^{\prime}\right)$, with significant fogging of the untreated sample, while the treated sample forms a continuous, non-diffracting water film. In addition, a slight anti-reflectivity (AR) is obtained due to the moth-eye effect ${ }^{36}$. This AR effect is quantified in Figure S1, where we see that single-sided nano-structured samples transmit almost $1 \%$ more visible light $(400 \mathrm{~nm}-800 \mathrm{~nm}$ ) than the unstructured ones. Later, we take advantage of the transmission properties, when conducting the optical transmission study of the dynamic water spreading on the model surface. The model surface is visualized using helium-ion nano-imaging in Figure $1 \mathrm{~h}$. We see that the nanostructures consist of rounded pillars ordered in a predominantly hexagonal array and have a height to diameter aspect ratio of about one. 
a

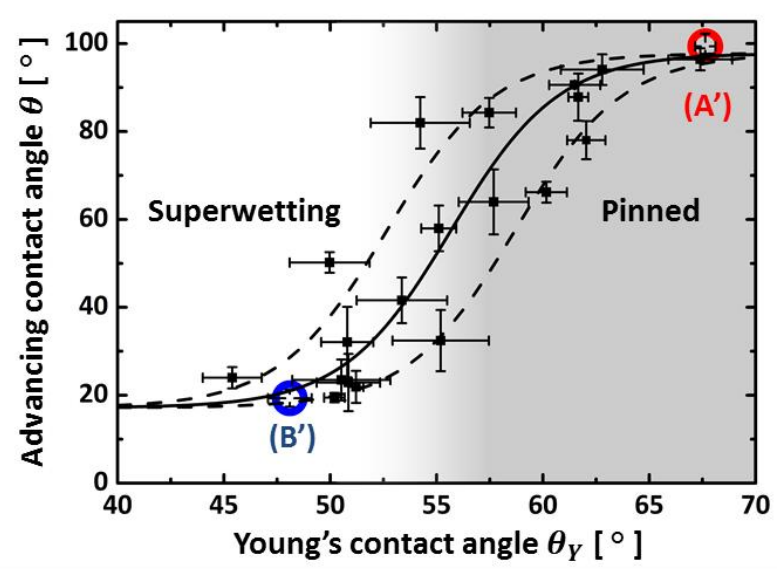

$21 \mathrm{sec}$.

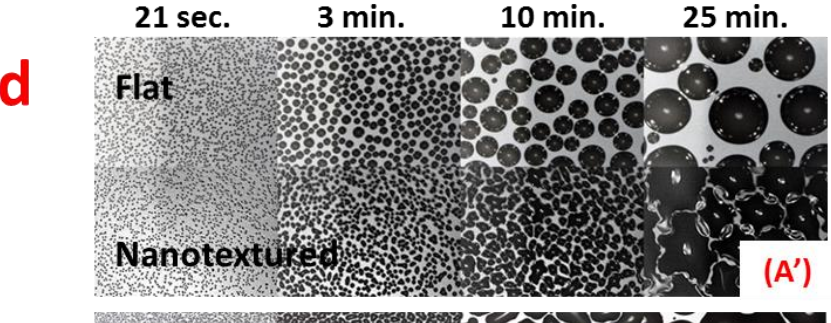

e

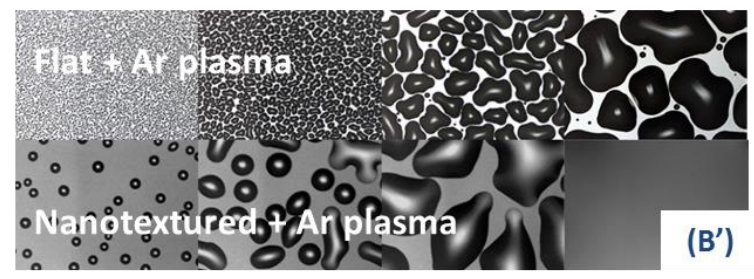

b Flat

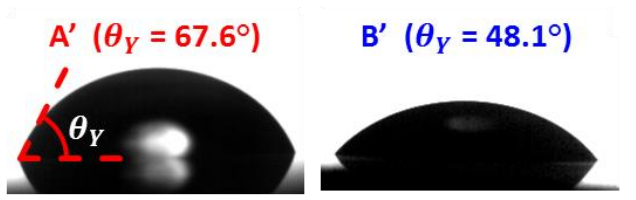

C Nanotextured

$A^{\prime}\left(\theta=99.4^{\circ}\right) \quad B^{\prime}\left(\theta=19.3^{\circ}\right)$

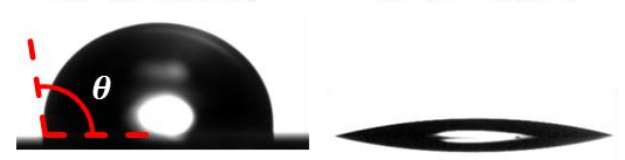

f

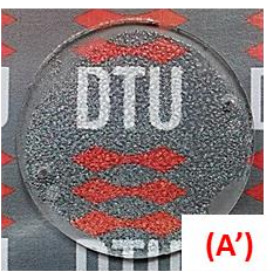

g

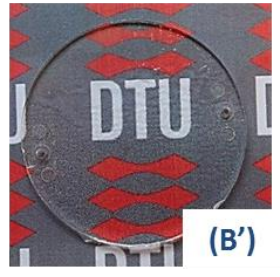

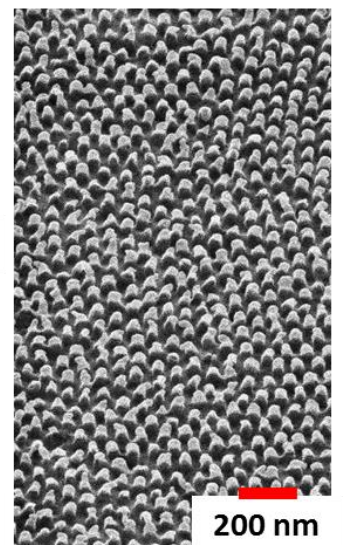

Figure 1. Experimental demonstration of criterion for spreading on textured, hydrophilic surface.

a: Advancing contact angles $\theta$ for nanostructured samples plotted vs their intrinsic contact

angle, $\theta_{Y} .$. The gray scale shading indicates the transition between the superwetting regime and

the pinning regime. The solid line represents a fit with a sigmoidal function to guide the eye, while

the dashed curves represent one standard deviation of the fitted threshold between the regimes.

b: Droplet profiles recorded on the flat (unstructured) samples. Data point $\mathbf{A}^{\prime}$ corresponds to an

untreated pristine sample with $\theta_{Y}=67.6^{\circ}$, while $\mathbf{B}^{\prime}$ is for a plasma treated sample with Young

contact angle $\theta_{Y}=48 \cdot 1^{\circ}$. c: Droplet profiles recorded on the nanostructured samples. Data point

$\mathbf{A}^{\prime}$ corresponds to an untreated sample having $\theta=99.4^{\circ}$, while $\mathbf{B}^{\prime}$ corresponds to a 
nanostructured and plasma treated sample with a contact angle $\theta=19.3^{\circ}$. d: Optical microscopy images recorded during condensation of water from saturated vapor while samples were cooled to $5^{\circ} \mathrm{C}$. Images are recorded at times as indicated. The images were obtained for untreated flat and nanostructured surfaces (condition $\mathbf{A}^{\prime}$ ), and in e: for treated (condition $\mathbf{B}^{\prime}$ ) flat and nanostructured surfaces as indicated. The width of each frame is roughly $1 \mathrm{~mm}$. f: Overview image of an untreated (condition $\mathbf{A}^{\prime}$ ) but nanotextured sample subjected to water vapor, and $\mathbf{g}$ : of a plasma treated (condition $\mathbf{B}^{\prime}$ ) and nanotextured sample. The samples measure $50 \mathrm{~mm}$ in diameter. h: Helium-ion nanoscopy image of $40^{\circ}$ tilted nano-pillar surface derived from BCP nanolithography and replicated in PMMA.

To understand these phenomena better, we investigated the surface chemistries in more detail, as summarized in Figure 2. Gradual changes to the surface chemistry were obtained by Ar plasma treatment. ${ }^{41-43}$ The primary effect is to render the samples more hydrophilic through an increase in surface free energy (Figure 2a; more details on the chemistry below).$^{42}$ For similar plasma activation and Ar pressure conditions, we found that Young's contact angle decreased approximately linearly with plasma treatment time (Figure S2). In addition, plasma treated PMMA has a hydrophobic recovery at the time scale of hours, allowing us to test the transition in the reverse direction (from hydrophilic to hydrophobic) shown in Figure $\mathbf{2 b}$. Indeed, a small increase in Young's contact angle from around $50^{\circ}$ to $55^{\circ}$ results in an abrupt change in the advancing contact angle for the nanostructured sample; $\sim 15^{\circ}$ to $60^{\circ}$. Hydrophobic recovery after plasma induced hydrophilization may result from hydrocarbon contamination, chain re-orientation, and outdiffusion of apolar moieties. ${ }^{43}$ However, unlike the coarse surface activation by the Ar plasma treatment, which relies on precise timing of the plasma process, we see that the hydrophobic recovery process is monotonic in time, and shows the return to the pinned state when the Young 
contact angle exceeds $\sim 50^{\circ}$ after 4-6 hours. Observation of the reverse transition corroborates our claim of a purely chemical change.

The PMMA surface hydrophilization was documented by X-ray photoelectron spectroscopy (XPS) measurements of pristine (Figure $\mathbf{2 c}$ ) and $70 \mathrm{~s}$ Ar plasma treated (Figure $\mathbf{2 d}$ ) PMMA surfaces. The plots show the decomposition of the spectral carbon 1 s peak into the four contributions $\mathbf{C} \mathbf{1}$ (C-C), C2 $(\mathrm{C}-\mathrm{H}), \mathbf{C} 3(\mathrm{C}-\mathrm{O})$, and $\mathbf{C 4}(\mathrm{C}(\mathrm{O})-\mathrm{O})$. It is found that the ratio $\mathbf{C 4 / C 3}$ is higher in Ar-treated samples (see Table S1). We interpret this as an introduction of acid groups to a fraction of the polymer units (Figure $\mathbf{2 e}$ ) by hydrolysis of ester groups as schematically shown in Figure $\mathbf{2 f}$. This substitution is the most likely cause for the significant hydrophilization, which is common during surface activation of polymeric materials. ${ }^{44}$ The soft Ar plasma treatment ${ }^{42}$ was chosen over the more aggressive $\mathrm{O}_{2}$ plasma ${ }^{45}$ in order to avoid the introduction of additional surface roughness. This is documented in Figure S3, where we compare AFM scans of unstructured PMMA samples before, and after the considerable plasma treatment for $70 \mathrm{~s}$. Indeed, the surface roughness of unstructured PMMA samples before and after was measured by AFM to be $R_{a}=(1.20 \pm 0.10) \mathrm{nm}$, and $R_{a}=(1.37 \pm 0.13) \mathrm{nm}$, respectively. 
a
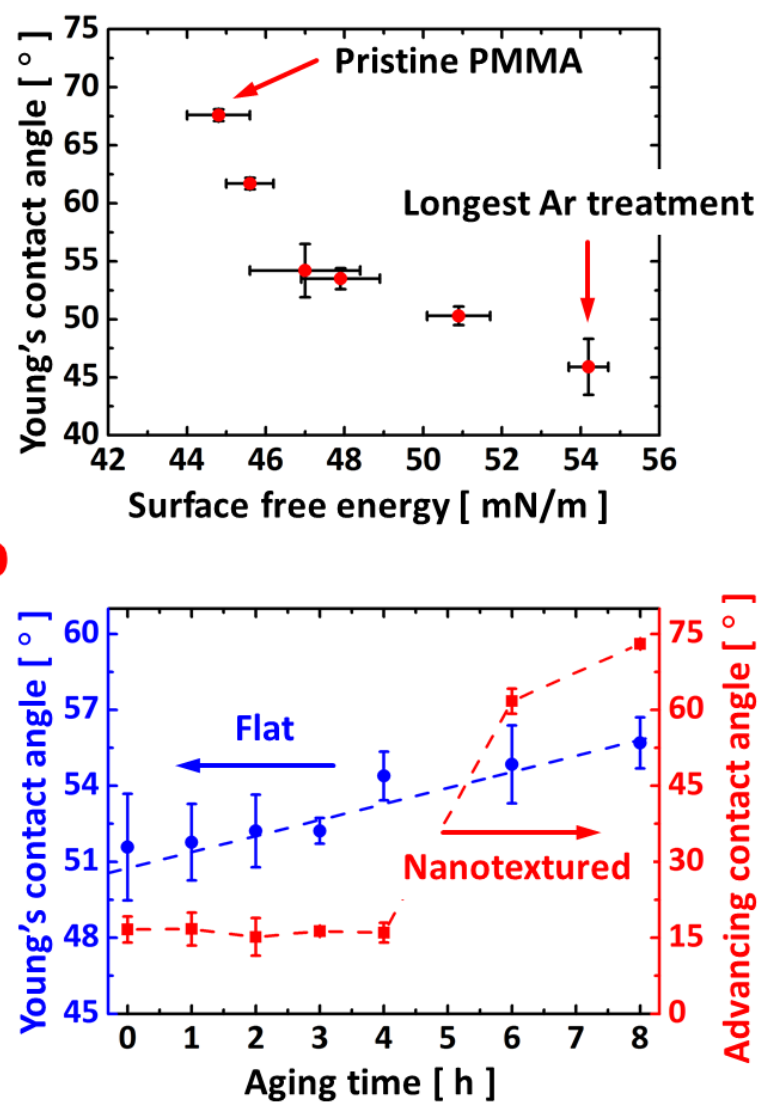
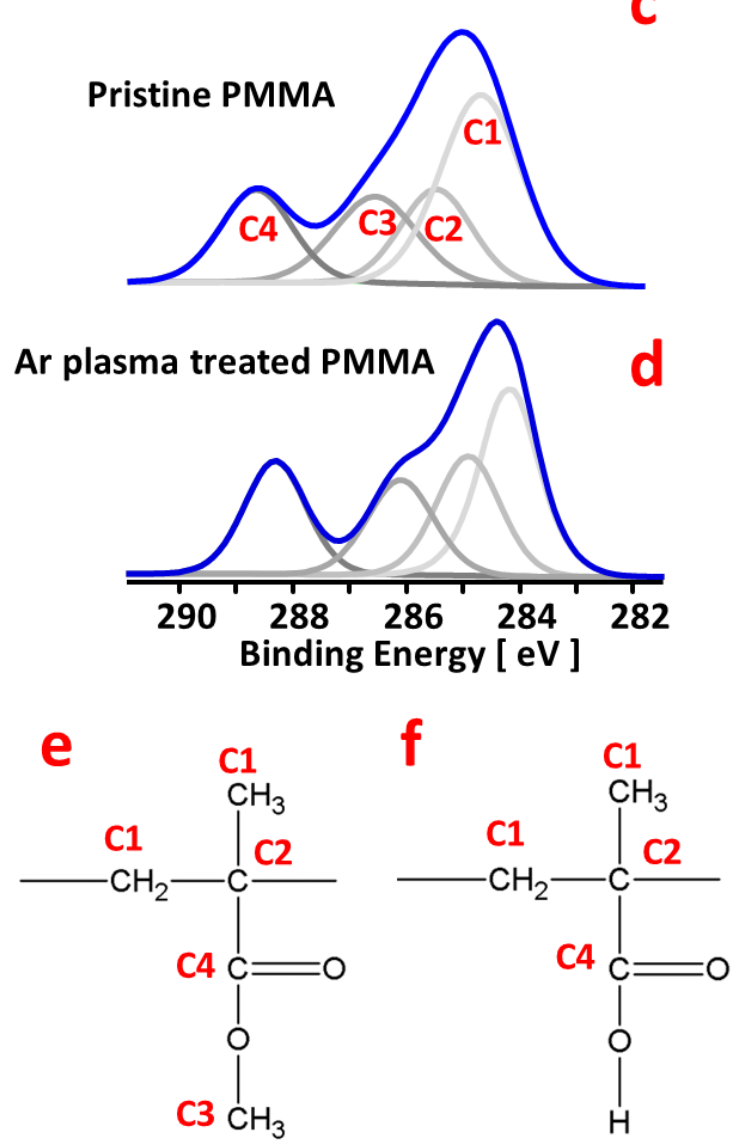

Figure 2. Experimental documentation of the plasma induced hydrophilization effect. a:

Dependence of PMMA surface free energy $\gamma_{s}$ on Young's contact angle $\theta_{Y}$ for a range of plasma treated samples together with the data for the pristine untreated sample. b: Measured advancing contact angles on $70 \mathrm{~s}$ Ar plasma treated PMMA surfaces during aging C: XPS spectrum of pristine PMMA surface. d: XPS spectrum of Ar plasma treated PMMA surface $\mathbf{e}:$ Chemical formula of pristine PMMA unit with specified carbon bonds $\mathrm{f}$ : Chemical formula of the modified units in the polymer surface upon Ar plasma treatment justifying the increased C4/C3 ratio in the C1s signal.

Evidently, the superwetting state of a macroscopic droplet (Figure 1a-c) and the ability to create a continuous film of condensate at micron level (Figure $\mathbf{1 d - g}$ ) are interconnected. To pinpoint what 
causes the relation; we observed the triple line for the two different states by transmission microscopy. In Figure $\mathbf{3}$ we present the droplet spreading experiments, where an example from each state is shown. The states again exhibit qualitatively different behavior with only the superwetting state supporting a hemiwicking flow. Figure 3a is a schematic of the transmission microscopy measurement, where we visualize the droplet spreading from below. Figure $\mathbf{3 b}$ shows data as obtained for selected times (raw videos for the presented time sequences are found in ESI, Video 1,2). Tracking the droplet front quantifies the spreading (see Figure 3c). For sample $\mathbf{A}$, the droplet spreading is slow, but propagates throughout the whole experiment. For sample $\mathbf{B}$, which was plasma treated for longer time than A, the droplet propagates about a factor of 20 faster, reaches a standstill in $<10$ seconds, and is then superseded by a hemiwicking film that initially continues with the same speed as the droplet was spreading, but soon after slows down. 
a

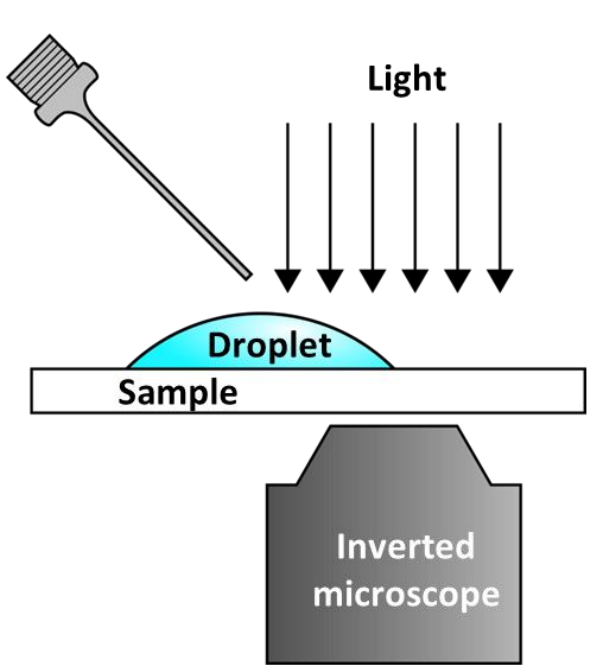

C

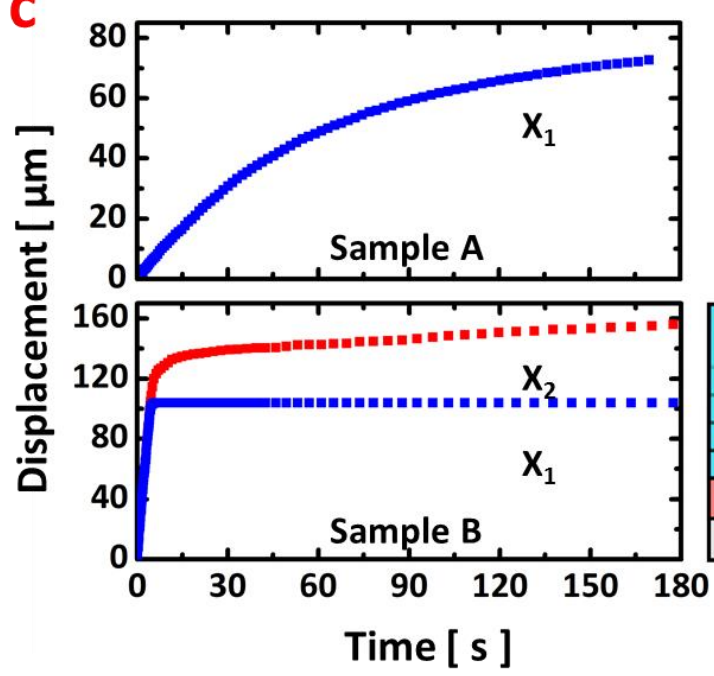

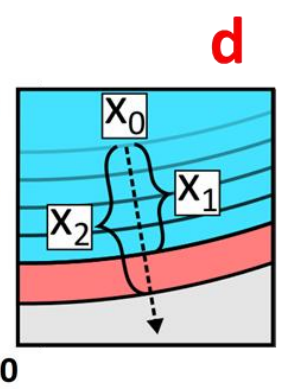

b
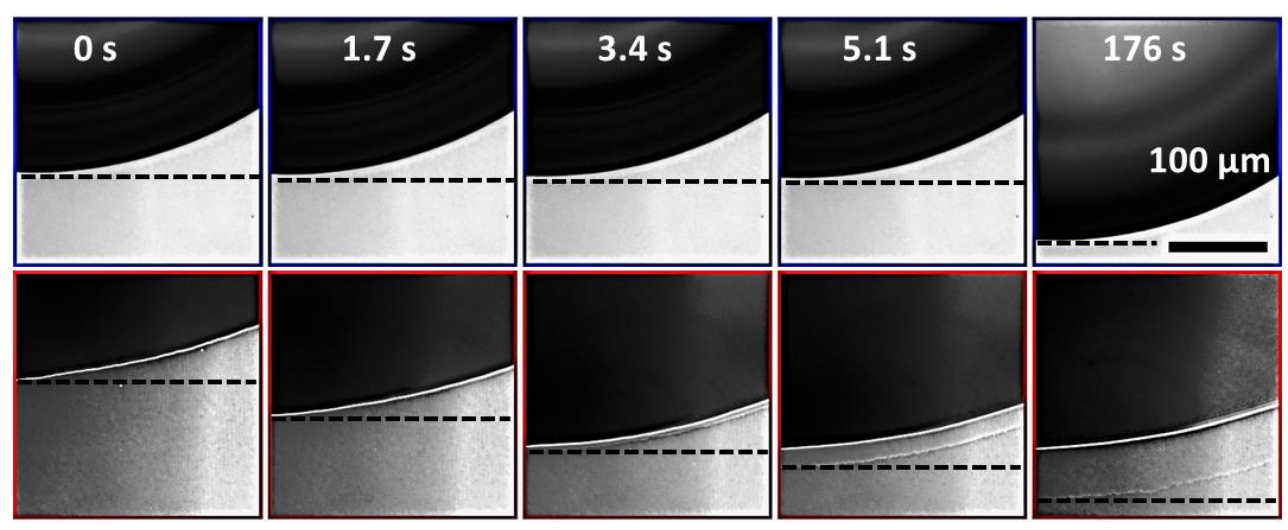

Sample A

$\theta_{Y} \approx 55^{\circ}$

$\theta=(70 \pm 2)^{\circ}$

\section{Sample B}

$\theta_{Y} \approx 45^{\circ}$

$\theta=(20 \pm 2)^{\circ}$

Figure 3. Recording of droplet spreading by transmission microscopy. a: Schematic of the

experimental setup comprising transmission optical microscopy using an inverted microscope.. b:

Image sequences from videos of the droplet evolution are shown after 0,1.7, 3.4, 5.1, and 176 sec.

The images show spreading of water on a surface in the pinned state (sample A), and in the superwetting state (Sample B) respectively. The dashed lines indicate positions of the waterfront at the given time. The advancing contact angle for sample $A$ was calculated to be $(70 \pm 2)^{\circ}$ and for sample B to be $(20 \pm 2)^{\circ}$. Estimates for Young's contact angles are also indicated. c: Extracted displacement of liquid front is plotted as a function of displacement time. For sample A, exhibiting pinning properties, the droplet front $x_{1}$ propagates but slows down gradually. $x_{0}$ indicates an 
arbitrary zero-position for the droplet front displacement. For Sample B, which supports the superwetting state, the droplet propagates much faster until it reaches a full stop after a few seconds. A hemiwicking film with front position $x_{2}$ continues the propagation after the droplet has reached a full stop. $\mathbf{d}$ : Schematic showing the definition of positions $x_{1}$ and $x_{2}$ with respect to the zero-position $x_{0}$.

The observed behavior contradicts the expectation based on thermodynamic arguments predicting increased wetting of hydrophilic surfaces when the roughness factor is increased. ${ }^{46}$ Although this prediction was shown experimentally for very rough nanograss glass surfaces having Young contact angles $\theta_{Y}$ below $30^{\circ},{ }^{47}$ we and many other groups find an unexpected increase in the apparent contact angle $\theta$ with increasing roughness ${ }^{48,49}$ owing to the pinning effect. ${ }^{22,50-54}$ Strong pinning can even cause droplets to spread along the underlying lattice directions creating polygonal droplet shapes. ${ }^{55}$ Despite these observations, the superwetting behavior of rough surfaces made from moderately hydrophilic materials, such as polymers, has only been sporadically investigated. ${ }^{11,13}$ The abnormally high contact angle, we attribute to the droplet being pinned in a metastable state away from thermodynamic equilibrium. However, zooming in on the triple line (as done in Figure 3) reveals that such pinned droplets, in fact, spread continuously; although very slowly. This dynamic motion is toward the thermodynamic equilibrium state through a series of metastable states. ${ }^{23,56}$ Wenzel theory predicts full wetting with contact angle $0^{\circ}$ above a critical Wenzel roughness factor, $r_{c}=1 / \cos \left(\theta_{Y}\right)$. Thus, for the roughness factor $\approx 2.5-$ 3 (see Supporting Information S5) estimated for our PMMA model surfaces, we would expect full wetting whenever Young's contact angle is below $66^{\circ}-70^{\circ}$. Instead, the transition to a small, but 
yet finite, advancing contact angle seems to occur only for a considerably lower Young contact angle of $\theta_{Y} \approx 50^{\circ}$; most clearly seen in the plot showing hydrophobic recovery in Figure $\mathbf{2 b}$.

The scientific literature on liquid front advancement on a solid surface is quite rich. For smooth surfaces, the droplet liquid front is typically found to follow Tanner's power law where it advances as $x(t) \propto t^{0.1} .{ }^{57,58}$ For hemiwicking flow on a pillar-built surface, Bico et al. ${ }^{59}$ found that the liquid front advanced with a power law $x(t) \propto t^{0.5}$, i.e. in analogy with the Washburn equation ${ }^{60,61}$ that considers capillary driven flow in a bundle of thin parallel tubes. Subsequently, a broader parameter space was explored proving a wider range of possible exponents for the power law. ${ }^{29,}$ 31, 62, 63 Our data follow a power law $x(t) \propto t^{\alpha}$, with $\alpha \approx 0.85-1.00$ for the first $\sim 10$ s after droplet launch (Figure S4a-c). This near-linear behavior is consistent with a pressure-driven, HagenPoiseuille flow of constant channel length. When a droplet reaches a full stop, a hemiwicking film emerge with an initial speed equal to that of the droplet before stopping (example on this seen in Figure 3c). Similar behavior was also observed and dubbed "synchronized spreading" by Kim et al. ${ }^{31}$ for a dense pillar array. For longer times, a slow-down is observed as a deviation from a straight line in the log-log plot Figure S4b, and in Figure S4e. We attribute this slow-down to evaporation effects. ${ }^{64}$ Regarding the superwetting state, we assigned a contact angle (see Figure S4f) to the droplet by measuring its radius after full stop and employing Equation $\mathbf{S 1}$. This analysis showed a clear correlation between contact angle and propagation speed of the hemiwicking film; the lower contact angle correlates with higher speed as expected for a Hagen-Poiseuille flow.

The driving force for the hemiwicking film is the uncompensated Laplace pressure, $\Delta p$, associated with the curved menisci at the liquid front. ${ }^{31,53,65,66}$ Thus, a permanently negative Laplace 
pressure allows hemiwicking. This Laplace pressure may be very high; 10-50 bar owing to the nanoscale dimensions of the surface texture. Thus, considerably higher than both the hydrostatic pressure $\rho g h_{\text {droplet }} \sim 10^{-4}$ bar and the Laplace pressure associated with the overall droplet curvature $\sim \gamma / R_{\text {droplet }} \sim 10^{-3}$ bar (where $\rho$ is the mass density, $g$ the acceleration of gravity, $h_{\text {droplet }}$ the height of the sessile droplet, and $R_{\text {droplet }}$ the radius of curvature of the sessile droplet). Further, we compare our droplet size with the capillary length $\ell_{c}=\sqrt{\rho g / \gamma}(\approx 2.7 \mathrm{~mm}$ for water). For droplet sizes somewhat smaller than $\ell_{c}$, gravity will have negligible influence on the droplet shape. In the present experiment, the droplet base radii are of order one millimeter and their heights even smaller, why we assume them to have shapes of spherical caps. Lastly, the governing dimensionless number for hemiwicking flow is the capillary number $C a=\eta V_{o} / \gamma$, where $V_{0}$ is the characteristic speed of advancement and $\eta$ the dynamic viscosity. The advancement speeds of the liquid fronts (visualized in Figure 3 ) are of the order $1-20 \mu \mathrm{m} / \mathrm{s}$ resulting in $\mathrm{Ca} \approx 1^{-}$ ${ }^{8}-10^{-7}$. It indicates a flow heavily dominated by surface tension over viscous forces. Consequently, we assume that the Laplace pressure associated with the curvature of the menisci is the only force and that it may be expressed in terms of a lateral and a vertical menisci radii of curvatures $R_{1}$ and $R_{2}$. In Equation (1) we attribute a minus sign to $R_{2}$ as positive curvature is considered from inside of the liquid (corresponding to a convex meniscus).

$$
\Delta p=\gamma\left(\frac{1}{R_{1}}-\frac{1}{R_{2}}\right)
$$

For a positive Laplace pressure the droplet will tend to become pinned, and only when capillary barriers locally break, the liquid front will advance to the next barrier. Assuming a perfect hexagonal array of cylindrical pillars, we elaborate further on this Laplace model in Supporting 
Information S6. The model corroborates onset of hemiwicking when Young's contact angle for the material gets low enough; below $\sim 50^{\circ}$ for our geometry. Regarding possible texture geometries, Semprebon et al. compared the wicking properties of square arrays of round and square pillars, and found stronger wicking properties for dense arrays of round pillars. ${ }^{67}$ In this respect, we emphasize that a hexagonal array of circular pillars is a generic closest packing geometry, and as we shall see shortly, only by decreasing the pitch to pillar diameter ratio $a / d$ can we make the structure denser, and thereby allow for a higher Young contact angle threshold for superwetting. However, the structures presented here are very close to the practical pillar density limit by having a gap between pillars of only $(a-d) \approx(18 \pm 2) \mathrm{nm}$, making it exceedingly difficult to increase the threshold value significantly. When below this limiting Young's contact angle, the hemiwicking film eventually overtakes the radial spread of the macroscopic droplet from which it originated. This occurs when the droplet reaches an equilibrium wetting state with no energetic benefits from further advancement. Here it rests on a composite surface comprised of dry pillar tops and liquid. ${ }^{59,65,68-70}$ The situation corresponds to a wetting state for a chemically heterogeneous surface obeying Cassie's law. ${ }^{46,71}$ Ishino and Okumura dubbed this state the "sunny-side-up" state, with the yolk representing the sessile droplet, and the white representing the wicked water film (Figure S7). ${ }^{68}$ In Figure S8, where the model and the data from Figure 1a are compared, we see that the model predicts an asymptotic value for low Young contact angles, i.e. for $\theta_{Y} \lesssim 50^{\circ}$, whereas for higher $\theta_{Y}$ the data begin to deviate substantially from the model. We interpret this deviation as a transition to a so-called pseudo-Wenzel state ${ }^{68}$, where the droplet "struggles" towards thermodynamic equilibrium, but never seems to get there (at least not during the observation time, which was 10 minutes for the longest lasting experiment). To the contrary, 
droplets in the superwetting state reach a full stop, which is a true characteristic of thermodynamic equilibrium.

The 4-6 hours to hydrophobic recovery for the plasma treated samples is ideal for fundamental studies but prohibitive for practical utilization such as for antifogging applications. Hence, we identified two different surface coatings that prolonged the lifetime of the superwetting effect considerably. Both coatings effectively make the pillar arrays denser by increasing pillar diameter with the coating thickness. In addition, one coating comprising $\sim 11 \mathrm{~nm} \mathrm{SiO}$ s slightly increased the roughness when applied to a flat surface, whereas another comprising $\sim 8 \mathrm{~nm}$ tungsten (W) coating (Figure S9) slightly decreased surface roughness. Arithmetic mean deviation $R_{a}$ roughness figures obtained by atomic force microscopy (AFM) are shown in Figure S10; $(1.70 \pm 0.12) \mathrm{nm}$ for $\mathrm{SiO}_{\mathrm{x}}$ and $(0.55 \pm 0.10) \mathrm{nm}$ for $\mathrm{W}$. The improved durability is presented in Figure 4 with the aging curves for the $\mathrm{W}$ coating in Figure 4a. During the first couple of days, the Young contact angle $\theta_{Y}$ increases quickly, but soon levels out to $40^{\circ}-45^{\circ}$ for a period of at least 56 days. Consequently, the surface remained in the superwetting state throughout the whole test period. The advancing contact angle stayed below $\sim 12^{\circ}$ and it exhibited superior anti-fogging behavior as demonstrated by the condensation experiments shown in Figures $\mathbf{4 b , c}$ (recorded after 56 days). For the $\mathrm{SiO}_{\mathrm{x}}$ coating, superwetting behavior lasted beyond the monitored period of 84 days. Similar to the W coating, the $\mathrm{SiO}_{\mathrm{x}}$ coating strongly influenced the hydrophilicity of the coated surfaces. However, it is well-known that hydrophilic surfaces tend to lose their hydrophilic property due to adhesion of hydrocarbons from the ambient air over time. ${ }^{72}$ For the $\mathrm{SiO}_{\mathrm{x}}$ coated nanotextured surfaces, the advancing contact angle remained stable for 14 days followed by a slight rise from $\lesssim 5^{\circ}$ to $13^{\circ}$. After 35 days, it had increased to $20^{\circ}$, where it remained stable for two months. Despite the 
increase, the superwetting behavior was preserved, as confirmed by the condensation experiment shown in Figure 4d,e. Eventually, after more than three months of aging (contact angle measured in the fourth month), this surface lost its superwetting property when the Young contact angle increased beyond $\sim 60^{\circ}$. A slightly higher crossover Young contact angle for $\mathrm{SiO}_{x}$ coated samples is expected, as compared to plasma treated samples, when taking the slightly larger interpillar aspect ratio into account. For the $\mathrm{W}$ coated samples, the Young contact angle remained below $50^{\circ}$ for at least four months. As an additional benefit, the nanotexture, further delays the aging effect of the attached $\mathrm{CH}$ bonds caused by environmental contamination, as the incoming diffusive flux of hydrocarbon groups is focused onto the apexes of pillars, ${ }^{71}{ }^{11}$ thereby shielding the more critical interpillar area from the contamination.

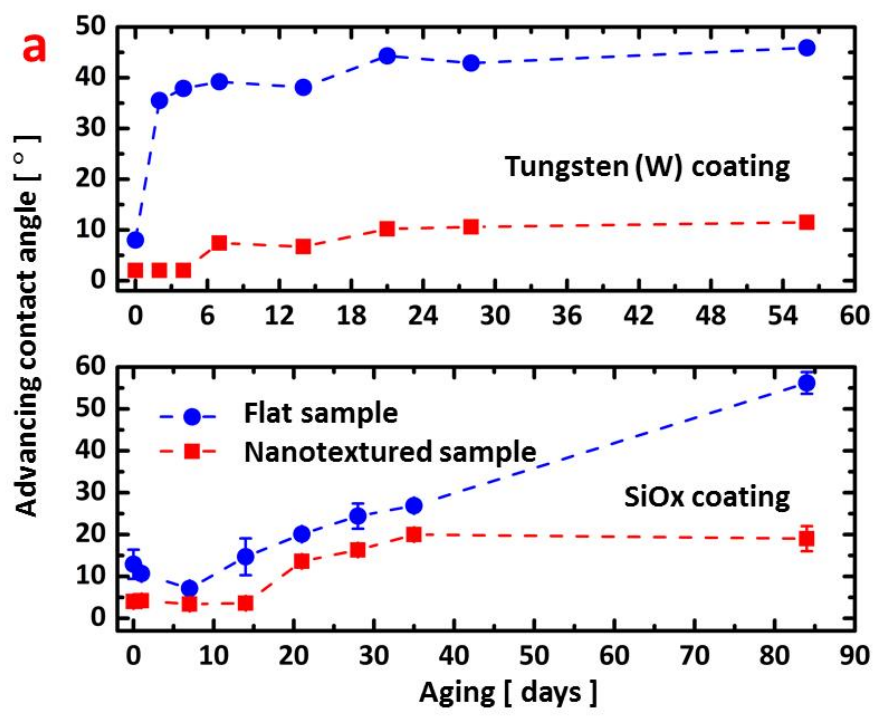

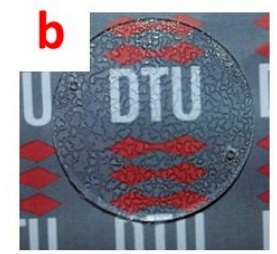

Flat $+\mathrm{W}$ w. water vapor

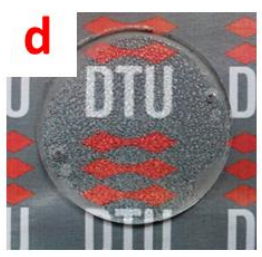

Flat + SiOx w. water vapor

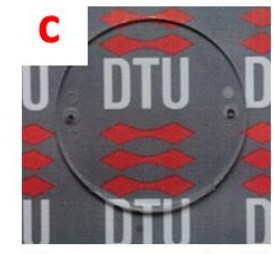

Nanotextured + W w. water vapor

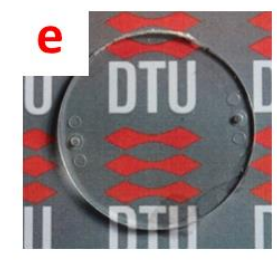

Nanotextured + SiOx w. water vapor

\section{f}

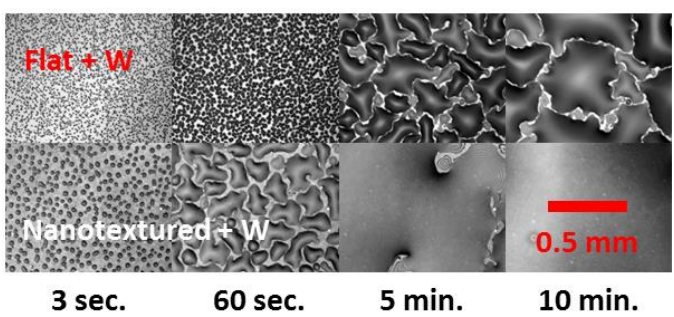

g

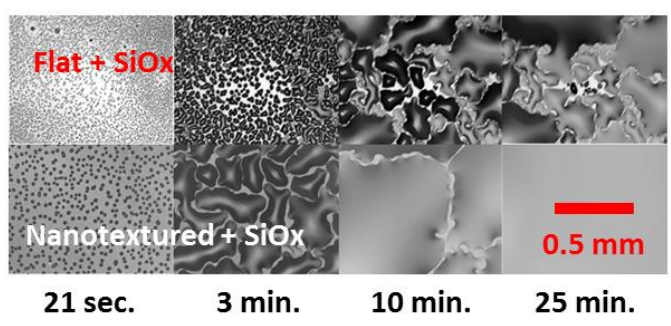


Figure 4. Aging and condensation experiments for thin-film coated samples. a: Measured advancing contact angles of $\mathrm{W}$ and $\mathrm{SiO}_{\mathrm{x}}$ coatings on both flat and nanotextured surfaces during aging. b-e: Overall images of samples subjected to water vapor; b: of flat with $\mathrm{W}$ coating, $\mathbf{c}$ : nanotextured with $\mathrm{W}$ coating, d: flat with $\mathrm{SiO}_{\mathrm{x}}$ coating, and e: nanotextured with $\mathrm{SiO}_{\mathrm{x}}$ coating. f: Optical microscopy images recorded during condensation of water from saturated vapor while samples were cooled to $5^{\circ} \mathrm{C}$. Images are recorded at times as indicated. The images were obtained for flat and nanostructured surfaces coated with W, and in $\mathbf{g}$ : for flat and nanostructured surfaces coated with $\mathrm{SiO}_{\mathrm{x}}$.

\section{Conclusion}

We employed block-copolymer self-assembly to fabricate optically transparent nanostructured surfaces in PMMA. The surfaces were subsequently exposed to varying degrees of soft argon plasma that enabled a study of the transition from a pinning state to a superwetting state for water. This transition was demonstrated through contact angle and water condensation studies. The transition to superwetting occurred when the material Young contact angle $\theta_{Y}$ dropped below $\sim 50^{\circ}-55^{\circ}$. Further, by employing transmission microscopy, we demonstrated that the superwetting state supports hemiwicking flow. The crossover in Young's contact angle was associated with a sign change of the Laplace pressure for the menisci of the liquid front when passing between pillars. Thus, surfaces having $\theta_{Y} \lesssim 50^{\circ}$ would support hemiwicking for the corresponding nanostructured surfaces, while surfaces having $\theta_{Y} \gtrsim 50^{\circ}$ would pin the droplets. The advancements of the macroscopic droplet front on both sides of the transition, and the 
displacement of the hemiwicking front was experimentally found to obey a power law $x(t) \propto t^{\alpha}$, with $\alpha \approx 0.94 \pm 0.05$, i.e. consistent with a predicted linear dependence in the limit for a dense pillar arrays by Kim et al. ${ }^{31}$ For surfaces in the superwetting state, the droplet spreading speed was up to a factor of $\sim 20$ larger than in the pinning state. Further, the superwetting state was shown to support a true thermodynamic equilibrium state for a droplet resting on a composite surface of dry pillar caps and the wicked water film in the voids. The apparent contact angle in this state corroborated the so-called "sunny-side-up" model of Ishino and Okumura ${ }^{68}$, where the yolk represents the sessile droplet, and the white the wicked liquid film.

Finally, two antifogging surfaces that did not suffer from fast aging caused by hydrophobic recovery were demonstrated. Hence, we tested the long term aging property for a thin $\mathrm{W}$ metal coating and a slightly thicker, dielectric $\mathrm{SiO}_{x}$ coating. Both coatings prolonged the lifetime of the superwetting state; at least four months for the $\mathrm{W}$ coating, and almost three months for the $\mathrm{SiO}_{\mathrm{x}}$. The W coating appears more stable of the two, and the better candidate for antifogging applications. To keep surfaces optically transparent, we recommend, using $\lesssim 10 \mathrm{~nm}$ W coating, as shown in Figure S11, where we see that a W coating of about $8 \mathrm{~nm}$ keeps the optical transmittance above $\sim 85 \%$ for a flat surface. The anti-reflection effect of the nanostructures will further compensate some of the optical loss due to the metal coating. The largest advantage of the metal coating over more involved hydrophilization strategies is its ease of manufacture by simple sputtering. W and PMMA are both approved and well-known materials for most applications. Moreover, the samples can be stored at room temperature and ambient pressure conditions without losing their superwetting property. 
In the absence of coatings, the desired superwetting property has a finite lifetime and needs reintroduction by Ar plasma, which is incompatible with most applications. However, we find that the class of temporarily existing materials obtained through plasma activation and characterized in Figure $2 \mathrm{a}$, seems to follow the general trend of conventional polymers regarding their Young contact angle dependence on surface free energy (Figure S12 and Table S3). This hints the material properties to be searched for in order to get permanent anti-fog by superwetting for noncomposite, recycle-friendly materials. Unfortunately, apart from exotic water-soluble polymers such as polyvinyl alcohol (PVOH), no common polymer has sufficiently low Young contact angle to support a superwetting state using our model texture. Hence, the engineering of surfaces exhibiting perpetual wetting relies on the development of a polymer system with a lower Young contact angle than currently existing ones.

In summary, our findings provide a deeper understanding of the surface properties required to support a superwetting state, and devise surface coatings that can be combined with the nanotexture for practical realizations of antifogging and haze-free polymeric windows. Their utilization span further than advanced optical elements such as lenses, mirrors, goggles, and wind shields; improved sunlight harvesting in photovoltaic devices; and better field visibility in endoscopy.

\section{Experimental Section}

Sample fabrication. The fabrication of master origination structures in $\mathrm{Si}$ is described in a recent publication by Telecka et al. ${ }^{38}$ The full process also comprising replication in polymer is depicted in Figure 5. 


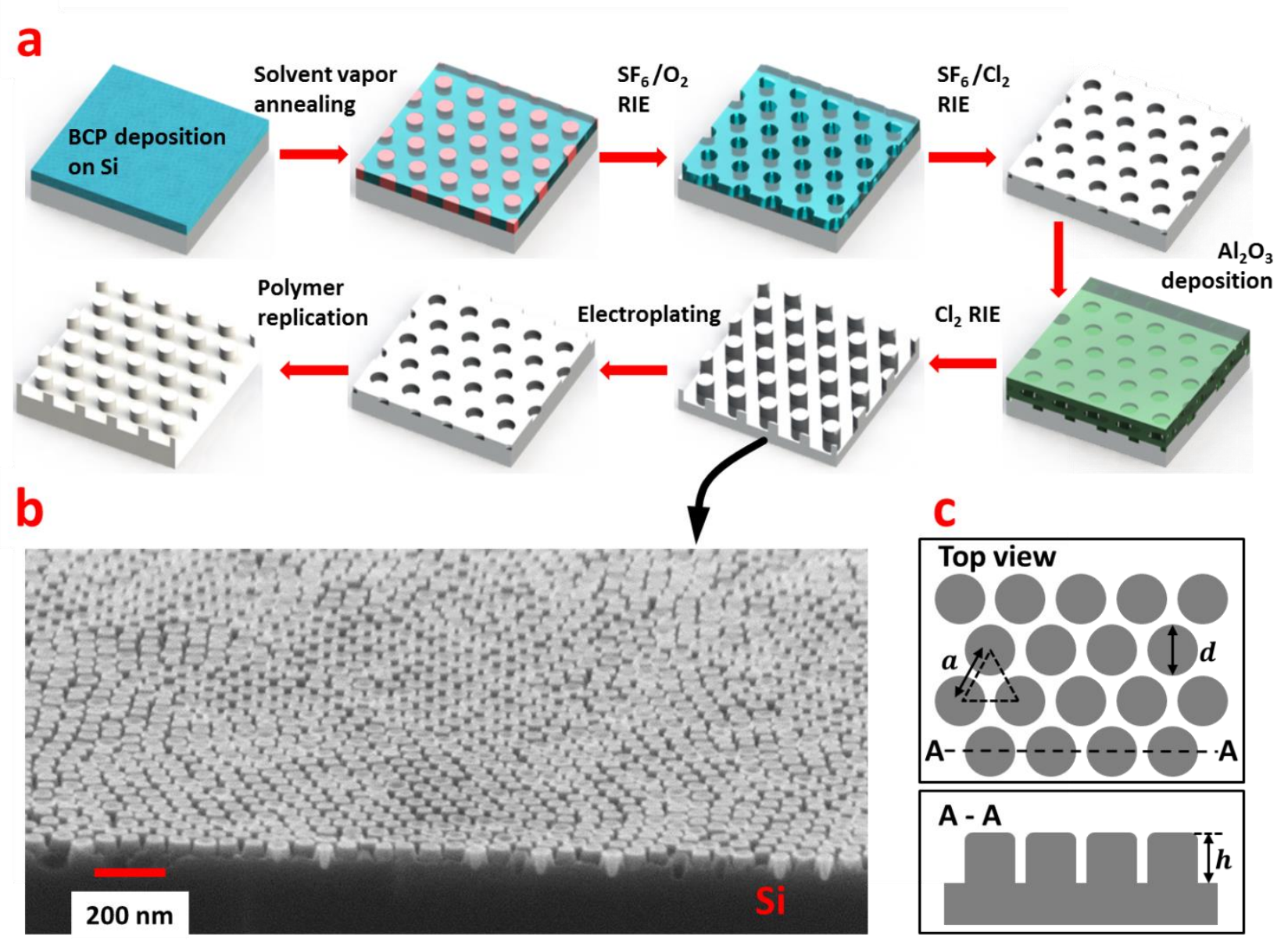

Figure 5. Fabrication of nanotextured samples templated by block-copolymer self-assembly. a:

Schematic workflow of the nanofabrication process to generate the nanotextured PMMA surface.

b: Scanning electron micrograph of cleaved Si master structure generated by BCP nanolithography.

c: Sketch of hexagonal pillar structure resulting from the fabrication process; top-view and profile

view.

Polystyrene - $b$ - polydimethylsiloxane PS - PDMS block - copolymer (SD24) with a number average molecular weight $(\mathrm{Mn})$ of $172 \mathrm{~kg} / \mathrm{mol}$ and a very narrow polydispersity index of PDI $\leq$ 1.06 (TableS1) was used for the nanolithography processing. SD24 was first dissolved in cyclohexane (0.25 wt \%) and then spin - cast (LabSpin6, süSS) on a single crystalline silicon (Si) wafer at a spinning speed of $2000 \mathrm{rpm}$, yielding a $28 \mathrm{~nm}$ thick film. SD24 was then subjected to the 
solvent annealing in methylcyclohexane at room temperature with the sample closed in a glass jar in the presence of the solvent vapor for 160 min, creating densely packed PS cylinders in a hexagonal array. Subsequent mask fabrication was conducted in an Advanced Silicon Etcher (STS MESC Multiplex ICP serial no. 30343). For pretreatment, SF $_{6}$ plasma was applied for $15 \mathrm{~s} \mathrm{(20} \mathrm{sccm,}$ 20 mTorr, $50 \mathrm{~W}$ of coil power and $0 \mathrm{~W}$ of platen power) to remove the PDMS residual layer created on a top of the annealed surface and subsequent $\mathrm{O}_{2}$ plasma etching for $11 \mathrm{~s}$ (10 sccm, 5 mTorr, $200 \mathrm{~W}$ coil power and $20 \mathrm{~W}$ of platen power) to remove PS blocks and oxidize PDMS blocks simultaneously, resulting in a hard silica mask of hexagonally orientated holes with high selectivity. Pattern transfer to the silicon was performed by reactive ion etching in ICP Metal Etch (SPTS serial no. MP0637) using a 35 second plasma breakthrough step (20 sccm SF, 3 mTorr, $100 \mathrm{~W}$ of coil power, and $10 \mathrm{~W}$ of platen power) followed by $20 \mathrm{~s}$ of $\mathrm{Cl}_{2}$ etching $(20 \mathrm{sccm} \mathrm{Cl}, 3 \mathrm{mTorr}, 300 \mathrm{~W}$ of coil power and $40 \mathrm{~W}$ of coil power) to create shallow holes. Nanopillars were fabricated through initial deposition of a $30 \mathrm{~nm} \mathrm{Al} \mathrm{O}_{3}$ mask layer on a patterned substrate by atomic layer deposition (ALD). ALD deposition was performed in Picosun ALD (model R200), using alternating exposures to trimethylaluminium (97\%, Aldrich) and deionized water $\mathrm{H}_{2} \mathrm{O}$ at $200^{\circ} \mathrm{C}$ for 300 cycles with $\mathrm{N}_{2}$ gas purge steps in between. The exposures and purge times for both precursors used in this study were $0.2 \mathrm{~s}$ and $25 \mathrm{~s}$, respectively. Adjustment of $\mathrm{Cl}_{2}$ etching time to $210 \mathrm{~s}$ (20 sccm, $3 \mathrm{mTorr}, 300 \mathrm{~W}$ of coil power and $40 \mathrm{~W}$ of coil power) allowed for tuning the nanotexture aspect ratio to 1 . The nanostructured silicon master was then sputter coated with a $\sim 85 \mathrm{~nm}$ NiV thin film (Custom System, Kurt J. Lesker), which acted as a seed layer for subsequent Ni electroplating (Microform 200 , Technotrans) of an inverted relief polarity metal mold with a thickness of $300 \mu \mathrm{m}$. The entire Si wafer, bonded to the mold, was removed by dissolving in a $10 \mathrm{M} \mathrm{KOH}$ (Sigma - Aldrich) bath at $80^{\circ} \mathrm{C}$. Before injection molding, the nickel insert was coated with a perfluorodecyltrichlorosilane 
(FDTS) self-assembled monolayer (MVD100E, Applied Microstructures Inc.) which worked as antistiction layer. ${ }^{73}$ Polymethyl methacrylate (PMMA, IG840, LG MMA Corporation, Korea) discs (50 $\mathrm{mm}$ diameter, $2 \mathrm{~mm}$ thick) were injection molded using an industrial injection molding machine (Victory 45, Engel, Austria) facilitated with the structured nickel mold. ${ }^{74}$ A variothermal molding process was employed with injection pressure 1000 bar, holding pressure 1200 bar and a melt temperature $250^{\circ} \mathrm{C}$. (Figure 5a).

Tuning of surface chemistry after replication. After replication, some of the flat and nanostructured PMMA surfaces were treated in a low - pressure argon plasma (Atto Plasma Cleaner, Diener Electronic) with conditions: $5 \mathrm{~W}, 60 \mathrm{~Pa}$ for $2-78 \mathrm{~s}$. The silica - based $\left(\mathrm{SiO}_{\mathrm{x}}\right)$ coating of PMMA surfaces was done by plasma enhanced chemical vapor deposition (PECVD). The deposition process was carried out in a custom build RF - powered capacitive coupled plasma reactor with a stainless steel reactor chamber, and a parallel plate electrode configuration where the sample holder was the ground bottom electrode, also described by Moreno-Couranjou et al. ${ }^{75}$ For the $\mathrm{SiO}_{x}$ coating, the plasma was fed with a hexamethyldisiloxane $\mathrm{HDMSO} / \mathrm{O}_{2}$ mixture with monomer and oxygen flow rates at $2 \mathrm{sccm}$ and $150 \mathrm{sccm}$, respectively. The layer thickness was controlled by the deposition time. Hence, the process was conducted at the power of $300 \mathrm{~W}$ and pressure of 300 mTorr for $48 \mathrm{~s}$, resulting in the $\sim 11 \mathrm{~nm}$ thick coating on both flat and nanotextured PMMA. XPS atomic fractions for this coating were determined to: $17 \% \mathrm{C}, 50 \% \mathrm{O}$, $23 \% \mathrm{Si}$. The tungsten (W) coating of PMMA surfaces, flat and BCP nanostructured, was done by employing a sputter coater (Cressington 208HR) at $80 \mathrm{~mA}$ current and pressure of $0.01 \mathrm{mbar}$ for $10 \mathrm{~s}$, resulting in the $\sim 8 \mathrm{~nm}$ thick coating on both flat and nanotextured PMMA. 
Morphological Characterization. SD24 film thickness was determined by a VASE Ellipsometer (J.

A. Woollam) at three different incident angles $\left(55^{\circ}, 60^{\circ}\right.$ and $\left.65^{\circ}\right)$. Nanoscopy images of the structured PMMA were taken by He - ion Zeiss Orion Microscope (Figure 1h) with very thin layer of $\mathrm{Au}-\mathrm{Pd}$ and a Field Emission Zeiss Supra 40VP Microscope (Figure 5b). The imaging reveals a predominantly hexagonally arranged array of pillars (Figure 5c). Atomic force microscopy (AFM) images were taken by an AFM NX20, Park Systems in non - contact mode with a tip of a radius < $12 \mathrm{~nm}$. 3D projections of scanned surfaces were analyzed in SPIP 6.2.2 software. Dimensions of nanopillars (diameter and pitch) were measured by Image $1.47 \mathrm{t}$ software.

Transmittance measurements. The transmission of the samples was measured using a Shimadzu UV-VIS 2600 spectrophotometer. The transmission percentages of the coated, flat, PMMA samples were measured vs. air in the wavelength range of $400-800 \mathrm{~nm}$. These measurements were used to document the anti-reflective behavior of the nano-structured samples (Figure $\mathbf{S 1}$ ) and to investigate the light transmission for the W coated samples (Figure S13). The corresponding thicknesses of the $\mathrm{W}$ layers were obtained by calibrating the thickness vs deposition time. This was done by deposition of $\mathrm{W}$ on flat Si wafers, and subsequently cleaving the wafers to measure the thickness by SEM.

Chemical Characterization. X-ray photoelectron spectroscopy (XPS) analysis of Ar plasma treated PMMA was carried out with a Thermofisher K-Alpha system using a monochromatic Al X-ray source at $1486.6 \mathrm{eV}$. A dual beam charge neutralization gun, working at $1 \mathrm{~V}$ with an emission current of $20 \mathrm{~mA}$, was used for charge neutralization. Survey $(-10-1350 \mathrm{eV})$ and high-resolution spectra ( $C 1 \mathrm{~s}, \mathrm{O} 1 \mathrm{~s}$ ) were acquired at a pass energy of $200 \mathrm{eV}$ and $50 \mathrm{eV}$, respectively, using a beam spot size of $400 \mu \mathrm{m}$. XPS analysis of the $\mathrm{SiO}_{x}$ coating was carried out with a PHI Versa Probe 
II X-ray spectrometer, using a monochromatic Al X-ray source at $1486.6 \mathrm{eV}$ operating at $100 \mathrm{~W}$. A dual beam charge neutralization gun, working at $1 \mathrm{~V}$ with an emission current of $20 \mathrm{~mA}$, was used for charge neutralization. Survey $(0-1200 \mathrm{eV})$ and high-resolution spectra (C 1s, O 1s, Si $2 p)$ were acquired at a pass energy of $117 \mathrm{eV}$ and $47 \mathrm{eV}$, respectively, using a spot size of $200 \mu \mathrm{m}$. In all cases, sample charging was corrected with respect to the position of the hydrocarbon component $\mathrm{C}-\mathrm{C}(\mathrm{H})$, in the $\mathrm{C}$ 1s signal, and analysis was typically achieved with a takeoff angle of $53^{\circ}$ with respect to the surface normal.

Numerical calculations. Numerical calculations to produce the modelling plots for the Laplace pressure model (S6) and the "sunny side up" model (S7) were done in PTC MathCAD Prime 4.0 by using the Solve Block feature of the software.

Contact angle measurements. Since the advancing contact angle is the contact angle for a surface that has not previously been wetted, and therefore is most reproducible, all measured contact angles were advancing contact angles measured instantly after the drop inflation was stopped. The advancing contact angles were measured by the inflation method (S10) using an optical tensiometer (Attension Theta optical tensiometer, Biolin Scientific Holding AB, Sweden) with a high - speed camera (3000 fps, MotionXtra N3, IDT, USA). Shapes of droplets were fitted by the Young - Laplace fitting method (Attention Theta software, version 4.2). For all contact angle measurements, droplets of $6.5 \mu \mathrm{l}$ volume were used. For each sample, three measurements were made at different spots of the sample and mean values with standard deviations were calculated. Contact angles of the superwetting samples were made on timescales much shorter than those associated with the hemiwicking flow. For plasma treated samples, the contact angle measurements were done within one hour from the plasma treatment. 
Determination of surface free energy. To obtain the surface free energy $\gamma_{s}$ for the PMMA surfaces we employed Fowkes method, ${ }^{76}$ which is widely used for the determination of the $\gamma_{s}$ of polymeric materials ${ }^{77}$. As the polar and dispersion liquids we used water $\left(\gamma_{l}=72.8 \mathrm{mNm}^{-1}\right)$ and diiodomethane $\left(\gamma_{l}=50.8 \mathrm{mNm}^{-1}\right)$, respectively.

Condensation measurements. Condensation experiments were carried out in a custom built environmental chamber $\left(5 \times 10 \times 10 \mathrm{~cm}^{3}\right)$, equipped with a thermoelectric cooler (gSKIN, XM 27 9C, Sibalco AG, Switzerland) enabling adjustment of the substrate temperature, a copper plate working as a sample holder, and a temperature probe clamped between those two. The backside of the transparent PMMA sample was made anti-reflective using a black permanent marker. The sample was attached to the copper plate using thermal paste, and the chamber was closed. Using a LabVIEW programmed PID controller, the temperature was set to $5^{\circ} \mathrm{C}$ and stabilized for $75 \mathrm{~s}$. Additional $8.75 \mathrm{~min}$ of stabilization was applied to assure the stabilization of the polymer sample. The relative humidity was continuously probed inside the chamber using a resistive hygrometer. The humidity of the vapor flow was set by choosing the mixing ratio between pure nitrogen and saturated DI water vapor prior to the chamber entrance. Diffusers were placed at both chamber inlet and outlet to remove any possible jet streams. Throughout the temperature stabilization time, $8 \mathrm{~L} / \mathrm{min}$ of nitrogen was continuously run through the chamber to keep the relative humidity at $3 \%$ and avoid any premature condensation. After temperature stabilization, $2 \mathrm{~L} / \mathrm{min}$ of DI water saturated vapor was introduced into the chamber and after 5 seconds the $8 \mathrm{~L} / \mathrm{min}$ of nitrogen was shut off. This caused a sudden increase in the temperature and humidity. The chamber humidity was approaching complete saturation in an exponential manner on a characteristic time scale of $36 \mathrm{~s}$. Images of the condensation process were taking throughout 25 minutes of an experiment in time intervals of 3, 15 and 60 s using a Zeiss AXIO ZOOM.V16 Optical Microscope. The 
condensation procedure was performed one to two times on flat surfaces, and two to three times on the nanotextured surfaces.

Transmission microscopy of droplet spreading. Droplet spreading dynamics were characterized using the inverted optical transmission microscope Zeiss Axio Observer A1. See also Søgaard et al. ${ }^{39} \mathrm{DI}$ water droplets of volumes $0.5,1.5$, and $3.0 \mu \mathrm{L}$ were pipetted using a motorized dispensing system controlled by Attention Theta software. The pendant droplet was slowly approached to the hydrophilic sample until it released from the needle. With a frame rate of $15.4 \mathrm{fps}$, a $383 \times 512$ $\mu \mathrm{m}^{2}$ area with pixel size $369 \mathrm{~nm}$ was captured for $3000-9000$ frames. For selected frames, the radial position of the droplet and the wick were determined using the open source software Tracker 4.9x. Each experiment had 3 different parts of the droplet tracked to assure method consistency. At the conclusion of each experiment, a top view image of the droplet was captured at lower magnification to allow for quantification of the equilibrium contact angle. The droplet radii were determined in MATLAB 2017b using the CircleFitByPratt method after an initial edge detection procedure using a standard deviation filter, wiener2 adaptive noise-removal, and threshold by Otsu's method.

\section{Acknowledgements}

We would like to thank Dr. Nis K. Andersen for the development of the injection moulding process used to replicate the nanotextured PMMA parts. In addition, we also thank Dr. Nis K. Andersen and Mr. Milan Laustsen for constructing the environmental chamber used for the experiments. The authors would also like to thank Dr. Anna V. Shneidman for helpful discussions and DTU Danchip for outstanding support in cleanroom processing and sample fabrication. AT and NKM would like to thank DTU Nanotech for granting their PhD stipends. The work was supported by 
Center for Nanostructured Graphene, CNG, Technical University of Denmark and by DTU Nanotech, Technical University of Denmark.

\section{Author Contributions}

AT, NKM and RT conceived the project and wrote the paper with input from all authors. TL and SN developed the BCP process. AT optimized the BCP process and fabricated the samples. NKM and AT conducted the Ar plasma treatments, the contact angle measurements, and analysed the data. NKM conducted the transmission microscopy measurements and analysed the data. AT characterized the samples by the SEM and AFM. JF and SC characterized the nanotextured PMMA samples by He-ion nanoscopy. FP performed the $\mathrm{SiO}_{\mathrm{x}}$ coating together with $\mathrm{AT}$ and $\mathrm{RdM}$. RdM and FP characterized the samples by XPS. AT discovered and characterised the W coating properties. AT and EL conducted the aging experiments. EL measured the optical transmittance of the W coated samples. RT and NKM developed the theory.

\section{Competing interests}

The authors declare no competing interests.

\section{Electronic Supplementary Information}

S1: Optical transmittance spectra for structured and flat samples; S2: Low-pressure Ar plasma activated PMMA surfaces; S3: Roughness of unstructured PMMA surfaces activated by Ar plasma; S4: Analysis of hemiwicking data; S5: Geometry of nano-surfaces; S6: Laplace pressure model; S7: The "sunny side up" wetting model; S8: Surface coatings; S9: Young's contact angle vs. surface free energy. Video 1, Spread of droplet in pinned state, real time video, $87 \mathrm{~s}, 383 \mu \mathrm{m} \times 512 \mu \mathrm{m}$; Video 
2, Spread of droplet and hemiwicking film in superwetting state, real time video, $42 \mathrm{~s}, 383 \mu \mathrm{m} \times$

$512 \mu \mathrm{m}$.

\section{References}

1. B. Bhushan and Y. C. Jung, Progress in Materials Science, 2011, 56, 1-108.

2. R. Blossey, Nature Materials, 2003, 2, 301-306.

3. M. Nosonovsky and B. Bhushan, Nano Letters, 2007, 7, 2633-2637.

4. V. Liimatainen, M. Vuckovac, V. Jokinen, V. Sariola, M. J. Hokkanen, Q. Zhou and R. H. A. Ras, Nature Communications, 2017, 8.

5. N. Miljkovic, R. Enright, Y. Nam, K. Lopez, N. Dou, J. Sack and E. N. Wang, Nano Letters, 2013, 13, 179-187.

6. Y. M. Hou, M. Yu, X. M. Chen, Z. K. Wang and S. H. Yao, Acs Nano, 2015, 9, 71-81.

7. N. K. Mandsberg and R. Taboryski, Langmuir, 2017, 33, 5197-5203.

8. L. Mishchenko, M. Khan, J. Aizenberg and B. D. Hatton, Advanced Functional Materials, 2013, 23, 4577-4584.

9. M. Nosonovsky and V. Hejazi, Acs Nano, 2012, 6, 8488-8491.

10. L. Mishchenko, B. Hatton, V. Bahadur, J. A. Taylor, T. Krupenkin and J. Aizenberg, Acs Nano, 2010, 4, 7699-7707.

11. R. Di Mundo, R. d'Agostino and F. Palumbo, Acs Applied Materials \& Interfaces, 2014, 6, 1705917066.

12. T. Mouterde, G. Lehoucq, S. Xavier, A. Checco, C. T. Black, A. Rahman, T. Midavaine, C. Clanet and D. Quere, Nature Materials, 2017, 16, 658-+.

13. J. Li, J. Zhu and X. F. Gao, Small, 2014, 10, 2578-2582.

14. Y. F. Huang, S. Chattopadhyay, Y. J. Jen, C. Y. Peng, T. A. Liu, Y. K. Hsu, C. L. Pan, H. C. Lo, C. H. Hsu, Y. H. Chang, C. S. Lee, K. H. Chen and L. C. Chen, Nature Nanotechnology, 2007, 2, 770-774.

15. S. Sunny, G. Cheng, D. Daniel, P. Lo, S. Ochoa, C. Howell, N. Vogel, A. Majid and J. Aizenberg, Proceedings of the National Academy of Sciences of the United States of America, 2016, 113, 1167611681.

16. A. T. Paxson and K. K. Varanasi, Nature Communications, 2013, 4.

17. E. Bormashenko, Advances in Colloid and Interface Science, 2015, 222, 92-103.

18. R. N. Wenzel, Industrial and Engineering Chemistry, 1936, 28, 988-994.

19. A. B. C. Cassie and S. Baxter Transactions of The Faraday Society, 1944, 40, 0546-0550.

20. S. Anand, A. T. Paxson, R. Dhiman, J. D. Smith and K. K. Varanasi, Acs Nano, 2012, 6, 10122-10129.

21. L. C. Gao and T. J. McCarthy, Langmuir, 2006, 22, 6234-6237.

22. D. Quere, M. J. Azzopardi and L. Delattre, Langmuir, 1998, 14, 2213-2216.

23. D. Cwikel, Q. Zhao, C. Liu, X. J. Su and A. Marmur, Langmuir, 2010, 26, 15289-15294.

24. H. Kim, S. R. Rao, E. A. Kapustin, L. Zhao, S. Yang, O. M. Yaghi and E. N. Wang, Nature Communications, 2018, 9.

25. A. K. Kota, G. Kwon, W. Choi, J. M. Mabry and A. Tuteja, Nature Communications, 2012, 3.

26. K. S. Liu, M. Y. Cao, A. Fujishima and L. Jiang, Chemical Reviews, 2014, 114, 10044-10094. 
27. X. J. Feng and L. Jiang, Advanced Materials, 2006, 18, 3063-3078.

28. G. McHale, N. J. Shirtcliffe, S. Aqil, C. C. Perry and M. I. Newton, Physical Review Letters, 2004, 93.

29. C. W. Extrand, S. I. Moon, P. Hall and D. Schmidt, Langmuir, 2007, 23, 8882-8890.

30. D. X. Ye, T. M. Lu and T. Karabacak, Physical Review Letters, 2008, 100.

31. J. Kim, M. W. Moon and H. Y. Kim, Journal of Fluid Mechanics, 2016, 800, 57-71.

32. C. K. Wemp and V. P. Carey, Langmuir, 2017, 33, 14513-14525.

33. H. Cho, H. Y. Kim, J. Y. Kang and T. S. Kim, Journal of Colloid and Interface Science, 2007, 306, 379385.

34. A. Tuteja, W. Choi, J. M. Mabry, G. H. McKinley and R. E. Cohen, Proceedings of the National Academy of Sciences of the United States of America, 2008, 105, 18200-18205.

35. M. R. Lotz, C. R. Petersen, C. Markos, O. Bang, M. H. Jakobsen and R. Taboryski, Optica, 2018, 5, 557-563.

36. P. B. Clapham and M. C. Hutley, Nature, 1973, 244, 281-282.

37. T. Li, Z. L. Wang, L. Schulte and S. Ndoni, Nanoscale, 2016, 8, 136-140.

38. A. Telecka, T. Li, S. Ndoni and R. Taboryski, Rsc Advances, 2018, 8, 4204-4213.

39. E. Søgaard, N. K. Andersen, K. Smistrup, S. T. Larsen, L. Sun and R. Taboryski, Langmuir, 2014, 30, 12960-12968.

40. T. Young, Phil. Trans. R. Soc., 1805, 95, 65-87.

41. M. Modic, I. Junkar, A. Vesel and M. Mozetic, Surface \& Coatings Technology, 2012, 213, 98-104.

42. R. X. Wang, Y. Shen, C. Zhang, P. Yan and T. Shao, Applied Surface Science, 2016, 367, 401-406.

43. K. Gotoh, Y. Kobayashi, A. Yasukawa and Y. Ishigami, Colloid and Polymer Science, 2012, 290, 10051014.

44. J. Zhao, L. J. Song, Q. Shi, S. F. Luan and J. H. Yin, Acs Applied Materials \& Interfaces, 2013, 5, 52605268.

45. K. Tsougeni, N. Vourdas, A. Tserepi, E. Gogolides and C. Cardinaud, Langmuir, 2009, 25, 1174811759.

46. G. Whyman, E. Bormashenko and T. Stein, Chemical Physics Letters, 2008, 450, 355-359.

47. T. Fujima, E. Futakuchi, T. Tomita, Y. Orai and T. Sunaoshi, Langmuir, 2014, 30, 14494-14497.

48. T. S. Meiron, A. Marmur and I. S. Saguy, Journal of Colloid and Interface Science, 2004, 274, 637644.

49. P. S. H. Forsberg, C. Priest, M. Brinkmann, R. Sedev and J. Ralston, Langmuir, 2010, 26, 860-865.

50. N. Anantharaju, M. V. Panchagnula, S. Vedantam, S. Neti and S. Tatic-Lucic, Langmuir, 2007, 23, 11673-11676.

51. J. L. Liu, Y. Mei and R. Xia, Langmuir, 2011, 27, 196-200.

52. N. K. Mandsberg and R. Taboryski, Surface Topography: Metrology and Properties, 2017, 5.

53. D. Kim, N. M. Pugno and S. Ryu, Scientific Reports, 2016, 6.

54. N. K. Mandsberg, O. Hansen and R. Taboryski, Scientific Reports, 2017, 7.

55. L. Courbin, E. Denieul, E. Dressaire, M. Roper, A. Ajdari and H. A. Stone, Nature Materials, 2007, 6, 661-664.

56. M. Kanungo, S. Mettu, K. Y. Law and S. Daniel, Langmuir, 2014, 30, 7358-7368.

57. P. G. Degennes, Reviews of Modern Physics, 1985, 57, 827-863.

58. L. H. Tanner, Journal of Physics D-Applied Physics, 1979, 12, 1473-\&.

59. J. Bico, C. Tordeux and D. Quere, Europhysics Letters, 2001, 55, 214-220.

60. E. W. Washburn, Physical Review, 1921, 17, 273-283.

61. J. C. Cai and B. M. Yu, Transport in Porous Media, 2011, 89, 251-263.

62. T. P. Allred, J. A. Weibel and S. V. Garimella, Langmuir, 2017, 33, 7847-7853.

63. T. T. Mai, C. Q. Lai, H. Zheng, K. Balasubramanian, K. C. Leong, P. S. Lee, C. Lee and W. K. Choi, Langmuir, 2012, 28, 11465-11471.

64. H. Hu and R. G. Larson, Journal of Physical Chemistry B, 2002, 106, 1334-1344. 
65. C. Q. Lai, T. T. Mai, H. Zheng, P. S. Lee, K. C. Leong, C. Lee and W. K. Choi, Physical Review E, 2013, 88.

66. C. Semprebon, S. Herminghaus and M. Brinkmann, Soft Matter, 2012, 8, 6301-6309.

67. C. Semprebon, P. Forsberg, C. Priest and M. Brinkmann, Soft Matter, 2014, 10, 5739-5748.

68. C. Ishino and K. Okumura, European Physical Journal E, 2008, 25, 415-424.

69. X. M. Xu, G. Vereecke, C. Chen, G. Pourtois, S. Armini, N. Verellen, W. K. Tsai, D. W. Kim, E. Lee, C. Y. Lin, P. Van Dorpe, H. Struyf, F. Holsteyns, V. Moshchalkov, J. Indekeu and S. De Gendt, Acs Nano, 2014, 8, 885-893.

70. M. Miwa, A. Nakajima, A. Fujishima, K. Hashimoto and T. Watanabe, Langmuir, 2000, 16, 57545760.

71. A. B. D. Cassie and S. Baxter, Transactions of the Faraday Society, 1944, 40, 0546-0550.

72. J. Drelich, E. Chibowski, D. D. Meng and K. Terpilowski, Soft Matter, 2011, 7, 9804-9828.

73. J. Cech and R. Taboryski, Applied Surface Science, 2012, 259, 538-541.

74. P. F. Ostergaard, J. Lopacinska-Jorgensen, J. N. Pedersen, N. Tommerup, A. Kristensen, H. Flyvbjerg, A. Silahtaroglu, R. Marie and R. Taboryski, Journal of Micromechanics and Microengineering, 2015, 25.

75. M. Moreno-Couranjou, F. Palumbo, E. Sardella, G. Frache, P. Favia and P. Choquet, Plasma Processes and Polymers, 2014, 11, 816-821.

76. F. M. Fowkes, Journal of Physical Chemistry, 1962, 66, 382-\&.

77. H. W. Fox and W. A. Zisman, Journal of Colloid Science, 1952, 7, 109-121. 